\title{
Lesões fatais em trauma numa grande metrópole brasileira: um estudo de autópsias
}

\section{Fatal trauma injuries in a brazilian big metropolis: a study of autopsies}

Jorge L. Wilson; Fernando A. M. Herbella, TCBC-SP²; Guilherme F. Takassi³; Danilo G. Moreno ${ }^{1}$; Ana C. Tineli ${ }^{1}$

\author{
RE S U M O
}

\begin{abstract}
Objetivo: Este estudo tem o objetivo revisar uma série de mortes por trauma em uma grande metrópole. A intenção é identificar as causas evitáveis de morte. Métodos: Foram estudados prospectivamente 500 casos não selecionados e consecutivos de morte associada ao trauma. As variáveis do estudo foram as seguintes: mecanismo do trauma, etiologia, local da morte, a intervenção cirúrgica, imperícia médica, órgãos lesados e prevenção da mortalidade. Os casos foram agrupados, segundo o mecanismo de trauma, em: trauma penetrante, trauma contuso, intoxicação, afogamento, queimadura e asfixia. Resultados: Foram abordados $418(83,6 \%)$ casos do sexo masculino e $82(16,4 \%)$ do sexo feminino (média de idade $39 \pm 19,6$ anos, variando de três a 91 anos). O trauma penetrante correspondeu a 217 (43\%) casos; já o trauma contuso representou $40 \%$ dos casos. O mecanismo de trauma mais comum de morte entre o trauma penetrante foi lesão por arma de fogo, representando $41 \%$ do total de casos. Dentro do conjunto dos traumas contusos, o mecanismo mais comum foi o de acidentes de transporte, o que representou $22 \%$ do total de óbitos. Aconteceram 71 (14\%) casos de mortes evitáveis: tromboembolismo em 35 (7\%); complicações infecciosas em 25 (5\%), imperícia médica em sete (1\%) e lesões tratáveis em pacientes não hospitalizados cinco (1\%). Conclusão: Este estudo mostra que a morte traumática, na cidade de São Paulo, está associada à lesões graves e complexas. Prevenção da morte está relacionada ao controle da violência.
\end{abstract}

Descritores: Ferimentos e lesões. Mortalidade. Autópsia. Prevenção e controle.

\section{INTRODUÇÃO}

$\mathrm{O}$ trauma é uma das principais causas de morte em todo o mundo, especialmente nas grandes cidades. São Paulo é a maior cidade da América Latina e a quarta cidade mais populosa do mundo, segundo a estimativa do United Nations World Urbanization Prospects. É habitada por 11.000 .000 de cidadãos que vivem em $1525 \mathrm{~km}^{2}$ que conduzem 5.300.000 automóveis e, também, possui os mesmos problemas encontrados nas grandes áreas urbanas, isto é, tráfego intenso e violência. O Trauma foi responsável por 7603 mortes em 2005, 3209 destas foram causados por homicídios e 1579 por acidentes de transporte. A primeira abordagem ao traumatizado é fornecida pelo serviço paramédico de São Paulo, criado há 15 anos. Ele é composto pelo Corpo de Bombeiros e equipado com 265 veículos de emergência (um para cada 41.500 habitantes e 5,7 $\mathrm{km}^{2}$ ), um helicóptero e quatro ambulâncias especiais equipadas com médicos e enfermeiros do trauma. O número de ocorrências (atendimento ao trauma e emergências clínicas) por ano supera 59.000 (161/dia) com um tempo médio de 13 minutos para chegar ao local do acidente.

O atendimento ao trauma é administrado a partir de sistemas regionais de trauma e centros que integram cuidados primários, secundários e terciários. Centros com serviços secundários e terciários são formados, em sua maioria, pelos hospitais de ensino público. O atendimento intra-hospitalar ao paciente traumatizado é desempenhado normalmente por uma equipe cirúrgica e não pelos grupos de medicina de emergência, como de praxe em outros países.

As leis brasileiras exigem autopsia de todos os casos de suspeita ou de morte não natural. O Instituto Médico Legal (IML) é um departamento da Polícia do Estado e é responsável por todas as autópsias forenses da cidade. O IML de São Paulo é composto por três necrotérios divididos de acordo com as regiões da cidade. Foram realizadas 6200 autópsias em $2001^{1}$ no instituto sede.

Trabalho realizado no Departamento de Cirurgia da Universidade Federal de São Paulo, São Paulo, Brasil.

1. Médico Residente do Departamento de Cirurgia Universidade Federal de São Paulo, São Paulo, Brasil; 2. Professor Afiliado, Livre-Docente do Departamento de Cirurgia da Universidade Federal de São Paulo, São Paulo, Brasil; 3. Aluno de Graduação do Departamento de Cirurgia do Universidade Federal de São Paulo, São Paulo, Brasil. 
Este estudo visa rever uma série de mortes por trauma em uma grande metrópole além de identificar as possíveis causas evitáveis de morte.

\section{MÉTODOS}

Um total de 500 casos não selecionados e consecutivos de mortes relacionadas com trauma, entre 2008 e 2009, foram estudados prospectivamente. Todos os cadáveres passaram por autopsia, na sede do IML, pelo mesmo médico legista. As variáveis do estudo foram: mecanismo de trauma, etiologia, local de morte (cenário ou hospital), intervenção cirúrgica, erro médico, órgãos lesados e morte evitável.

Os casos foram agrupados segundo o mecanismo de trauma em: trauma penetrante, trauma contuso, intoxicação, afogamento, queimadura e asfixia. Trauma penetrante foi classificado baseado na etiologia de arma de fogo ou arma branca. Trauma contuso foi classificado baseado nas etiologias relacionadas com os acidentes de transporte, queda e agressão. A intervenção cirúrgica foi definida como qualquer procedimento cirúrgico, independentemente da complexidade, incluindo acessos vasculares por dissecção e drenagens torácicas.
Os erros terapêuticos ou as lesões diagnosticadas de forma inadequada foram definidos como erro médico.

A morte foi considerada evitável quando indivíduos que sofreram lesões tratáveis (traumatismo na ausência de: acometimento de grandes vasos, do coração ou da medula; de graves danos cerebrais, ou asfixia), porém não foram hospitalizados, associada ao erro médico; a morte devido à complicações infecciosas e a morte devido a eventos tromboembólicos (Figura1).

\section{RESULTADOS}

Foram $418(83,6 \%)$ casos do sexo masculino e $82(16,4 \%)$ do sexo feminino. A média etária foi de 39 $\pm 19,6$, mediana 35, variando de três a 91 anos. A distribuição dos casos de acordo com os mecanismos de trauma, etiologia, local da morte, intervenção cirúrgica, os órgãos feridos e o número de mortes evitáveis é descrito na tabela 1 . O número médio de tiros por índividuo vítima de ferimento por arma de fogo foi de 4,24 (871 tiros/205 casos). Um total de 386 (77,2\%) pacientes foram hospitalizados e 114 (22,8\%) morreram no local do acidente.

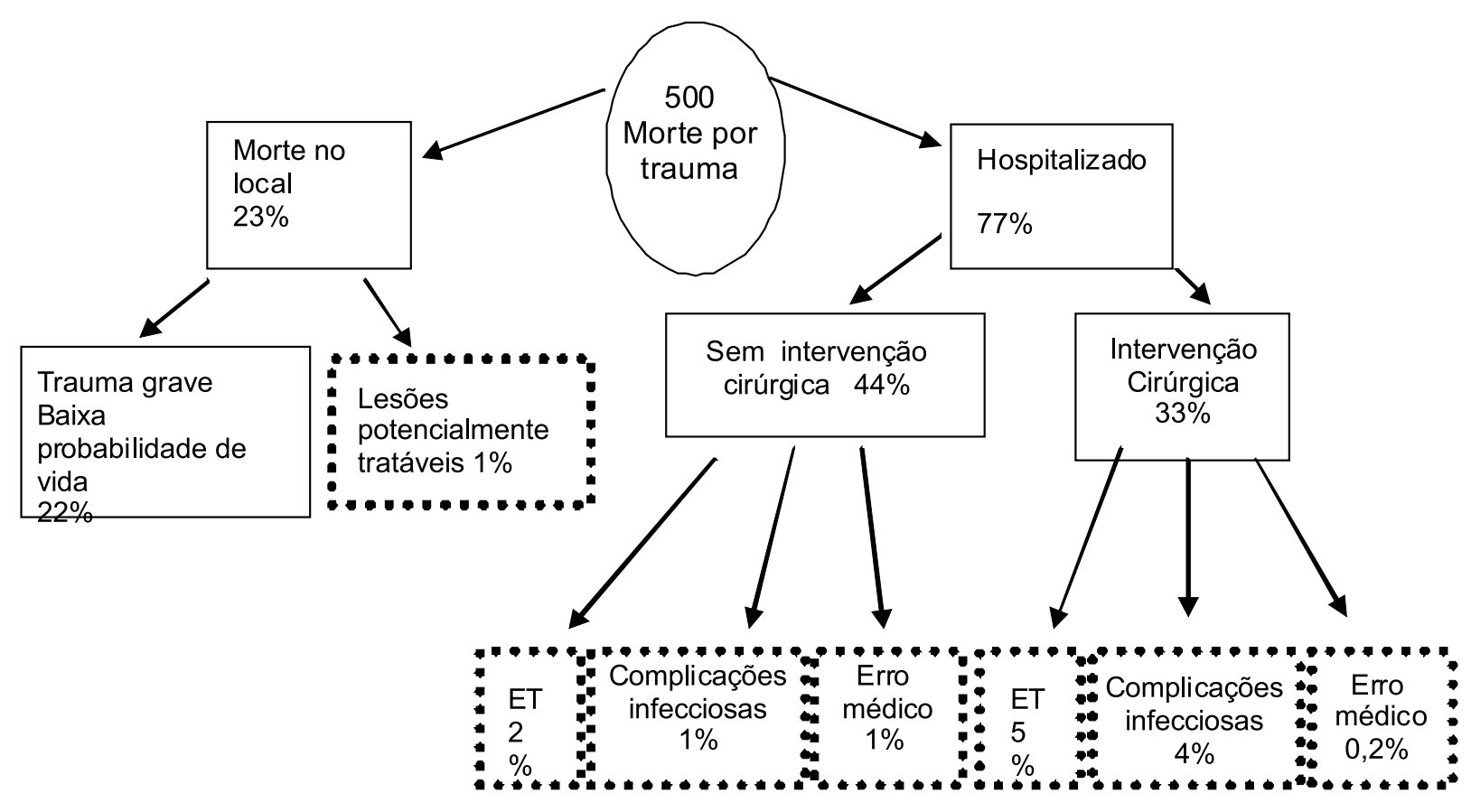

$\because \because:$ mortes evitaveis $\quad$ ET $=$ eventos tromboembolicos

Figura 1 - Fluxograma indicando prevenção de trauma-mortalidade relacionada. 
Tabela 1 - Distribuição dos casos de acordo com o mecanismo de trauma ( $n=500)$.

\begin{tabular}{|c|c|c|c|c|c|}
\hline Mecanismo* & Causa* & $\begin{array}{l}\text { Morte no } \\
\text { local ** }\end{array}$ & $\begin{array}{l}\text { Intervenção } \\
\text { cirúrgica* * }\end{array}$ & $\begin{array}{l}\text { Orgãos } \\
\text { lesados }\end{array}$ & Evitáveis* \\
\hline \multirow{13}{*}{$\begin{array}{l}\text { Penetrante } \\
217(43,4)\end{array}$} & Arma de fogo & \multirow[t]{8}{*}{$49(23,9)$} & \multirow[t]{8}{*}{$47(22,9)$} & Cérebro - 103 & $11(2,2):$ \\
\hline & $205(41,0)$ & & & Pulmão - 107 & 4 erros médicos \\
\hline & & & & Membros - 70 & 3 complicações infecciosas \\
\hline & & & & Coração - 60 & 3 lesões tratáveis \\
\hline & & & & Fígado - 48 & 1 tromboembolismo \\
\hline & & & & Intestinos - 28 & \\
\hline & & & & Estômago - 21 & \\
\hline & & & & Baço - 9 & \\
\hline & Arma branca & \multirow[t]{5}{*}{$4(33,3)$} & \multirow[t]{5}{*}{$5(41,6)$} & Pulmão - 5 & $2(0,4)$ \\
\hline & $12(2,4)$ & & & Coração - 2 & 1 lesões tratáveis \\
\hline & & & & Fígado - 2 & 1 complicações infecciosas \\
\hline & & & & Baço - 1 & \\
\hline & & & & Estômago - 1 & \\
\hline Contuso & Transporte & \multirow[t]{7}{*}{$16(14,2)$} & \multirow[t]{7}{*}{$63(56,2)$} & Cérebro - 83 & $17(3,4):$ \\
\hline \multirow[t]{14}{*}{$199(39,8)$} & $112(22,4)$ & & & Membros - 46 & 12 tromboembolismo \\
\hline & & & & Fígado - 40 & 5 complicações infecciosas \\
\hline & & & & Pulmão - 27 & \\
\hline & & & & Baço - 27 & \\
\hline & & & & Coração - 9 & \\
\hline & & & & Intestinos - 5 & \\
\hline & Queda & \multirow[t]{5}{*}{$7 \quad(9,2)$} & \multirow[t]{5}{*}{$41(53,9)$} & Cérebro - 53 & $25(5,0)$ \\
\hline & $76(15,2)$ & & & Membros - 19 & 15 Tromboembolismo \\
\hline & & & & Fíigado - 5 & 7 complicações infecciosas \\
\hline & & & & Pulmão - 4 & 3 erros médicos \\
\hline & & & & Baço - 3 & \\
\hline & Assalto & \multirow[t]{3}{*}{$2(18,1)$} & \multirow[t]{3}{*}{$2(18,1)$} & Cérebro - 9 & - \\
\hline & $11(2,2)$ & & & Baço - 2 & \\
\hline & & & & Fígado - 1 & \\
\hline \multirow{11}{*}{$\begin{array}{l}\text { Outros } \\
84(16,8)\end{array}$} & Queimadura & \multirow[t]{3}{*}{$1 \quad(9,0)$} & \multirow[t]{3}{*}{$5(45,4)$} & $\longrightarrow$ & $8(1,6):$ \\
\hline & $11(2,2)$ & & & & 5 tromboembolismo \\
\hline & & & & & 3 complicações infecciosas \\
\hline & Intoxicação & \multirow[t]{2}{*}{$1 \quad(6,6 \%)$} & \multirow[t]{2}{*}{0} & \multirow[t]{4}{*}{--} & $2(0,4)$ \\
\hline & $15(3,0 \%)$ & & & & 2 complicações infecciosas \\
\hline & Asfixia & \multirow[t]{2}{*}{$6(75,0)$} & \multirow[t]{2}{*}{$1(12,5)$} & & $1(0,2)$ \\
\hline & $8(1,6)$ & & & & 1 complicação infecciosa \\
\hline & Afogamento $4(0,8)$ & \multirow[t]{4}{*}{$2(50,0)$} & \multirow[t]{4}{*}{0} & \multirow[t]{4}{*}{--} & - \\
\hline & Outros $46(9,2)$ & & & & $5(1,0):$ \\
\hline & & & & & 2 tromboembolismo \\
\hline & & & & & 3 complicações infecciosas \\
\hline
\end{tabular}

Valores representados em (\%)

* \% em relação ao conjunto da população $(n=500)$

* \% em relação a causa

As intervenções cirúrgicas foram realizadas em 167 (43,3\%) das pessoas hospitalizadas. Aconteceram 71 (14\%) casos por mortes evitáveis (Tabela 2). Erro médico foi identificado em sete casos, todos devido à falta de diagnóstico adequado: cinco (hemotórax) e dois (hematoma extradural). As lesões tratáveis em vítimas que não foram hopitalizadas se apresentaram com um caso por arma branca atingindo o pulmão e três casos de arma de fogo: 1) projétil no fígado, 2) dois projéteis, lesão em intestino delgado e 3) quatro projéteis, ferindo pulmão, intestino delgado.

\section{DISCUSSÃO}

Autópsia como ferramenta para avaliação do trauma 
Tabela 2 - Causas evitáveis de morte.

\begin{tabular}{lcc}
\hline Causas & $\mathrm{n}$ & \% do total $(\mathrm{n}=500)$ \\
\hline Tromboembolismo & 35 & 7,0 \\
Complicações infecciosas & 25 & 5,0 \\
Imperícia médica & 7 & 1,4 \\
Lesões tratáveis em pacientes não hospitalizados & 4 & 0,8 \\
Total & 71 & 14,2 \\
\hline
\end{tabular}

Os estudos através da autópsia de casos de trauma representam uma ferramenta valiosa para uma nobre revisão, um controle de qualidade e, por fim, um melhor manejo clínico desses pacientes ${ }^{2,3}$. Estudos mostraram que um número significativo de lesões não localizadas inicialmente é apenas detectado na autópsia, com dados variando de 11 a $22 \%{ }^{4,5}$. Não só em relação às lesões diagnosticadas, mas também a associação à gravidade do trauma avaliada pelo Injury Severity Scale (ISS) é diferente quando os dados da autópsia são comparados com os achados clínicos ${ }^{3,6}$.

Apesar da importância da autopsia para avaliação do trauma, a literatura internacional revela que não mais que $50 \%$ dos pacientes falecidos devido ao trauma são autopsiados 5,7 . Em São Paulo, a autopsia é obrigatória em todos os casos de morte não natural.

Nossos resultados demonstram a importância dos exames necroscópicos, uma vez que, 71 mortes de 500 casos eram potencialmente evitáveis.

\section{Epidemiologia do trauma em São Paulo}

Nossos resultados mostraram que o trauma tem afetado, especialmente, os jovens do sexo masculino, seguindo os padrões internacionais.

Foi observado um equilíbrio entre trauma penetrante $(43,4 \%)$ e trauma contuso $(39,8 \%)$, com ferimentos por arma de fogo ( $41 \%$ ) e acidentes de transporte $(22 \%)$ como os principais mecanismos de trauma. Distribuições diferentes de mecanismos de trauma foram encontradas em diferentes cidades ao redor do mundo com base em problemas sociais locais. Por exemplo, em um estudo norueguês ${ }^{8}$, que avaliou 260 autópsias de trauma e mostrou uma incidência de $87 \%$ de trauma contuso, $31 \%$ devido aos meios de transporte e $25 \%$ associado às quedas; apenas $13 \%$ dos casos corresponderam a trauma penetrante. Em Auckland, Nova Zelândia, a principal causa de morte traumática foi enforcamento (36\%), seguido por acidentes com meios de transporte (32\%) e quedas $(10 \%)^{9}$.

No Brasil, Fraga et al. ${ }^{10}$, analisaram quase 2000 autópsias por trauma no município de Campinas. Os autores mostram uma média etária ainda mais jovem do que na nossa casuística (28 anos) com, também, preponderân- cia de ferimentos complexos, sendo que trauma torácico esteve presente em metade dos casos.

A complexidade das lesões encontradas em nosso estudo é notável. A maioria dos casos representa traumatismo cranioencefálico grave e lesão de múltiplos órgãos. Um quarto dos casos morreu no local e, mais de metade das vítimas que tiveram assistência hospitalar, não foram operadas. A maioria dos casos de trauma é abordada com intervenções cirúrgicas devido aos seguintes fatos: 1) o acesso cirúrgico vascular é muitas vezes necessário, 2) trauma torácico é tratado com drenagem pleural ou toracotomia na maioria das vezes e 3 ) trauma abdominal é, em grande parte dos casos, conduzido através de laparotomia. Nós acreditamos que os pacientes não foram operados nos hospitais devido à desanimadora expectativa de sobrevida quando da chegada em grande parte dos casos.

\section{Prevenção de Mortalidade}

Como dito anteriormente, a morte por trauma na cidade de São Paulo é representada por traumas graves e complexos. O percentual de mortes potencialmente evitáveis é aparentemente maior quando comparado com outros estudos ${ }^{2,11}$. No entanto, uma comparação direta não pode ser feita, uma vez que a maioria dos estudos é baseada apenas em pacientes internados ${ }^{2}$. Além disso, a definição de morte evitável é variável, adaptada às realidades locais e aos dados disponíveis. Bok Yoo et al. ${ }^{12}$, através de grande estudo restrospectivo em mais de 5000 autópsias, mostram que o tromboembolismo pulmonar fatal está associado ao trauma.

A deficiência do nosso estudo é a falta de informações clínicas, devido às limitações do Instituto Médico Legal. Além disso, a comparação com traumas não letais não foi feita.

Este estudo mostra que a morte por trauma, na cidade de São Paulo, está associada à lesões graves e complexas. A maioria das causas evitáveis de morte foram o tromboembolismo e as complicações infecciosas, porém, o número de mortes evitáveis através do tratamento médico é pequeno. Tais fatos sugerem que a prevenção das mortes deve ser conquistada através do controle da violência. 


\section{A}

Objective: This study aims to review a series of deaths by trauma in a large metropolis. The intention is to identify preventable causes of death. Methods: We prospectively studied 500 unselected and consecutive cases of death associated with trauma. The study variables were: mechanism of injury, etiology, site of injury, surgical intervention, medical malpractice, damaged organs and the prevention of mortality. The cases were grouped according to the mechanism of injury in: penetrating trauma, blunt trauma, poisoning, drowning, burns and suffocation. Results: We examined 418 (83.6\%) males and 82 (16.4\%) females (mean age $39 \pm$ 19.6 years, ranging from three to 91 years). Penetrating trauma accounted for 217 (43\%) cases, while blunt trauma accounted for $40 \%$ of cases. The most common mechanism of injury in death by penetrating trauma was gunshot, representing $41 \%$ of cases. Within the set of blunt trauma, the most common mechanism was traffic accident, which represented $22 \%$ of total deaths. There were 71 (14\%) cases of preventable deaths: thromboembolism in 35 (7\%), infectious complications in 25 (5\%), medical malpractice in seven (1\%) and treatable lesions in outpatients in five (1\%). Conclusion: This study shows that traumatic death in the city of São Paulo is associated with serious and complex injuries. Prevention of these types of death would be related to the control of violence.

Key words: Wounds and injuries. Mortality. Autopsy. Prevention and control.

\section{REFERÊNCIAS}

1. Herbella FA, Fernandes PH, Delmonte C, Del Grande JC. Forensic autopsy costs in the city of São Paulo. São Paulo Med J 2003; 121(3):139-42.

2. Gruen RL, Jurkovich GJ, McIntyre LK, Foy HM, Maier RV. Patterns of errors contributing to trauma mortality: lessons learned from 2,594 deaths. Ann Surg 2006; 244(3):371-80.

3. Martin BT, Fallon WF Jr, Palmieri PA, Tomas ER, Breedlove L. Autopsy data in the peer review process improves outcomes analysis. J Trauma 2007; 62(1):69-73.

4. Sharma BR, Gupta M, Bangar S, Singh VP. Forensic considerations of missed diagnoses in trauma deaths. J Forensic Leg Med 2007; 14(4):195-202.

5. Sharma BR, Gupta M, Harish D, Singh VP. Missed diagnoses in trauma patients vis-à-vis significance of autopsy. Injury 2005; 36(8):976-83.

6. Marx WH, Simon HM, Jumbelic M, Sposato E, Nieman G. Severity of injury is underestimated in the absence of autopsy verification. J Trauma 2004; 57(1):46-9.

7. Mushtaq F, Ritchie D. Do we know what people die of in the emergency department ? Emerg Med J 2005; 22(10):718-21.

8. Søreide K, Krüger AJ, Várdal AL, Ellingsen CL, Søreide E, Lossius HM. Epidemiology and contemporary patterns of trauma deaths: changing place, similar pace, older face. World J Surg 2007; 31(11):2092-103.

9. Pang JM, Civil I, Ng A, Adams D, Koelmeyer $\mathrm{T}$. Is the trimodal pattern of death after trauma a dated concept in the 21st century ? Trauma deaths in Auckland 2004. Injury 2008; 39(1):102-6.
10. Fraga GP, Heinzl LR, Longhi BS, Silva DC; Fernandes Neto FA, Mantovani M. Trauma cardíaco: estudo de necropsias. Rev Col Bras Cir 2004; 31(6):386-90.

11. Davis JW, Hoyt DB, McArdle MS, Mackersie RC, Eastman AB, Virgilio RW, Cooper G, Hammill F, Lynch FP. An analysis of errors causing morbidity and mortality in a trauma system: a guide for quality improvement. J Trauma 1992; 32(5):660-5; discussion 6656.

12. Bok Yoo HH, Mendes FG, Alem CER, Fabro AT, Corrente JE, Queluz TT. Achados clinicopatológicos na tromboembolia pulmonar: estudo de 24 anos de autópsias. J Bras Pneumol 2004; 30(5):426-32 .

Recebido em 19/02/2010

Aceito para publicação em 22/04/2010

Conflito de interesse: nenhum

Fonte de financiamento: nenhuma

\section{Como citar este artigo:}

Wilson JL, Herbella FAM, Takassi GF, Moreno DG, Tineli AC. Lesões fatais em trauma em uma grande metrópole brasileira: um estudo de autópsias. Rev Col Bras Cir. [periódico na Internet] 2011; 38(2). Disponível em URL: http://www.scielo.br/rcbc

\section{Endereço para correspondência:}

Fernando A. M. Herbella

E-mail: herbella.dcir@epm.br 